\title{
Genetic engineering to improve quality, productivity and value of crops
}

Abhaya M. Dandekar $\quad$ Neal Gutterson

Over the next 25 years, we believe that the most significant changes in crops will come about by applying genetic engineering tools. Crops may be bioengineered to produce modified kinds of starch, oils and high-value proteins for better nutrition, medical diagnostics and industrial uses. For example, walnuts and peanuts containing healthier oils, along with oxidative stability, could become available to consumers. Seedless vegetables and other fruits should appear in the marketplace within 10 years. Oil-producing seed crops may be modified to create specialty oils for a variety of nonfood products such as detergents, lubricants, inks and dyes. Feed seeds engineered to produce higher concentrations of sulfurcontaining amino acids could improve wool growth in sheep. Plants could be modified to deliver oral vaccines that prevent diseases such as hepatitis and influenza. Strawberries are being targeted by genetic engineering to extend their shelf life, and within 25 years fields may be planted with varieties that allow farmers to control the timing of fruit production. Although currently controversial, we believe genetic engineering will prove to be invaluable to the future improvement of agricultural systems. To enhance the competitiveness of California agriculture, government, university scientists and industry must work together to ensure the application of genetic engineering tools to improve crops.

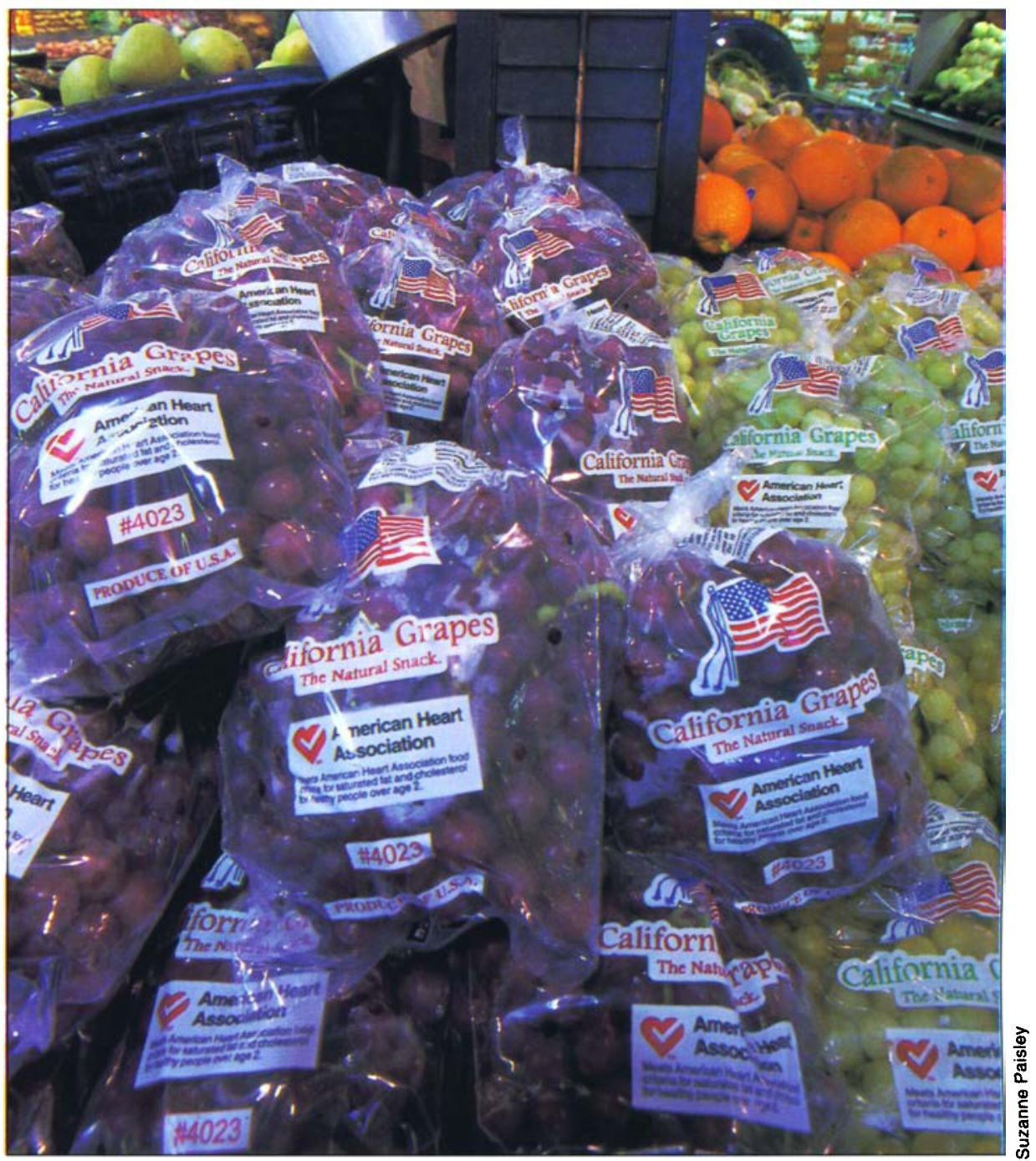

Seedless grapes, made possible by conventional breeding techniques, are popular with consumers. Scientists are exploring genetic-engineering strategies to bring new seedless fruits and vegetables to market more quickly.

$\mathrm{T}$ wo prominent features of agriculture in the 20th century have been the use of breeding and genetics to boost crop productivity and the use of agricultural chemicals to protect crops and enhance plant growth. Against the dire predictions of Thomas Malthus in 1798 , this integration of chemicals and plant breeding have resulted in food sufficiency and variety, helping to meet the needs of an ever-increasing population (Chrispeels and Sadava 1994).

In the 21st century, crops must produce good yields while conserving land, water and labor resources. At the same time, consumers expect plants to improve their health and provide necessary nutrients. Integrating conventional technologies with molecular biology and genomics could make all this possible, as genetic resources to 


\section{As the tools of genomics and genetic engineering are applied, we envision lettuce that does not flower in the fields, earlier-flowering strawberries and easier production of day-neutral strawberries.}

improve plants will be derived from the ongoing effort to discover, isolate and characterize genes that encode agriculturally important traits (Martin 1998).

Many, if not most, of the changes California consumers can expect in produce will be achieved through the application of genetic engineering. Although currently controversial, we believe genetic engineering will be invaluable to the future improvement of agricultural systems. In this article we describe current and future concepts under active investigation by universitybased and private researchers in the United States and worldwide. We chose several examples that we feel come to fruition during the next 25 years, but it is likely that there will be other unanticipated discoveries with profound implications for agriculture. Research to discover the function of genes that underlie both simple and complex traits is well on its way and greatly expanding, aided by new genomics and bioinfomatics (computational analysis of large biological data sets) research tools.

\section{Quality improvements}

Plants are remarkable in their capacity to synthesize a variety of organic substances, such as vitamins, sugars, starches and amino acids. As many as 80,000 different substances are synthesized in plants, including macronutrients and micronutrients significant to human health (Dellapenna 1999). Many of these substances, however, are undesirable "anti-nutrients" which can be detrimental to human health.

We expect that genomics will bolster plant biochemistry as researchers seek to understand the metabolic pathways for the synthesis of these compounds. Identifying rate-limiting steps in synthesis could provide targets for genetically engineering biochemical pathways to produce augmented amounts of compounds and new compounds. Targeted expression will be used to channel metabolic flow into new pathways, while gene-silencing tools will reduce or eliminate undesirable compounds or traits.

Carbohydrates. Plants manufacture both polymeric carbohydrates, like starch, and individual sugars, like sucrose. Plant starch is used in a wide range of industrial applications such as coatings for paper and textiles and as a gelling agent in the food industry (Heyer et al. 1999). It is now possible to make starches that are free of the amylose fraction, resulting in a gelling agent that is clearer and forms a gel at a lower temperature. Starches with higher levels of amylose are more desirable as coating agents and maintain texture at higher temperatures. For example, "sticky rice" has lower amylose content. The presence or absence of amylose greatly influences the physiochemical properties of starch; genetic engineering will result in specialized starches with higher value for specific applications.

In the paper industry, starches sometimes must be chemically modified by phosphorylation, in which phosphate mono-ester residues are chemically added to corn starch. Some starches (e.g., potato) are naturally phosphorylated, containing the enzymes to add this residue to the starch molecule. Cloning the gene responsible for phosphorylation of glucans makes it possible to engineer precise levels of phosphorylation, resulting corn starch that is more useful to the paper industry.

The chain length and distribution in amylopectin, the more highly structured (branched) component of starch, can be manipulated by altering the starch synthase activity. Genes respon- sible for the synthesis of fructans can modify plants of agronomic value to produce this polymeric carbohydrate (Heyer et al. 1999).

Fructans are an important ingredient in "functional" foods because they promote health and help to reduce the risk of colon cancer. Sugar beets that accumulate higher levels of fructans could be developed as a source for low-calorie sweeteners.

Lipids, fats and oils. Dietary fats and oils contribute to balanced nutrition, providing energy, fat-soluble vitamins and the essential polyunsaturated fatty acids (linoleic and $\alpha$ linolenic) required for growth, cellmembrane function, development and disease prevention. Conversely, fats and oils have been implicated in a diverse group of ailments such as obesity, cancer and heart disease.

A whole range of genetically modified oil seeds may be available in the future to promote health and prevent disease. In addition, specialty seeds with novel oil composition may be available to serve unique nutritional needs. For example, plants could be engineered to generate Lorenzo's Oil, a mixture of longchain, saturated fatty acids used to treat patients suffering from the inherited genetic disorder ALD (adrenoleukodystropy).

Changing the composition of plantbased fats and oils can also improve their taste, quality and oxidative stability. Rancidity, a common off-flavor, can be reduced by regulating the amount of polyunsaturated fats or through the accumulation of antioxidants such as vitamin E, which preserves fats and oils and prevents oxidation.

For example, walnuts or peanuts could be modified to maintain healthy oils and oxidative stability. Natural margarines could be made in plants through the synthesis of saturated fatty acids like those found in cocoa butter. This would not only improve the taste but also make it possible to produce margarine free of the unhealthy trans fatty acids that accumulate in partially hydrogenated traditional margarines. 
In addition, oil-producing seed crops may be modified to make specialty oils for a variety of nonfood products such as soaps, detergents, cosmetics, candles, lubricants, grease, polymers, plasticizers, coatings, inks, printing, textile dyes and surfactants (Topfer et al. 1995). Unlike their petroleum counterparts, these products would be biodegradable and better for the environment. The economic value of plants modified for pharmaceutical, medical or industrial applications will be much greater.

Proteins. Plant proteins provide amino acids important for human health. Many plant proteins, like those present in corn seed, do not have the complete complement of essential amino acids. Plant-seed proteins can be modified to express proteins with a more desirable amino-acid composition.

This is particularly important for animal feeds, where seeds engineered to produce a higher concentration of sulfur-containing amino acids could improve wool growth in sheep. The amino-acid composition of seed-storage proteins found in seeds, nuts, fruits and tubers can be modified to enhance nutritional value.

Plants may also be modified to produce proteins that aid in mineral nutrition, such as hemoglobin to improve iron uptake and other specific proteins to improve calcium uptake.

A significant segment of the U.S. population suffers from food allergies, an immunoglobulin (IgE)-mediated hypersensitivity reaction to particular plant proteins. Understanding the structure of the antigenic determinants of proteins that contribute to severe and often life-threatening allergy reactions could lead to the development of plant products that alter these proteins, greatly limiting the problem. Care must be taken in assessing transgenic products for their allergenic potential.

Nutritional components. Healthconscious consumers are compelling farmers and seed companies to improve the overall nutritional quality of their products. Extensive medical, biochemical and epidemiological research points to specific plant-produced substances (phytochemicals), as well as

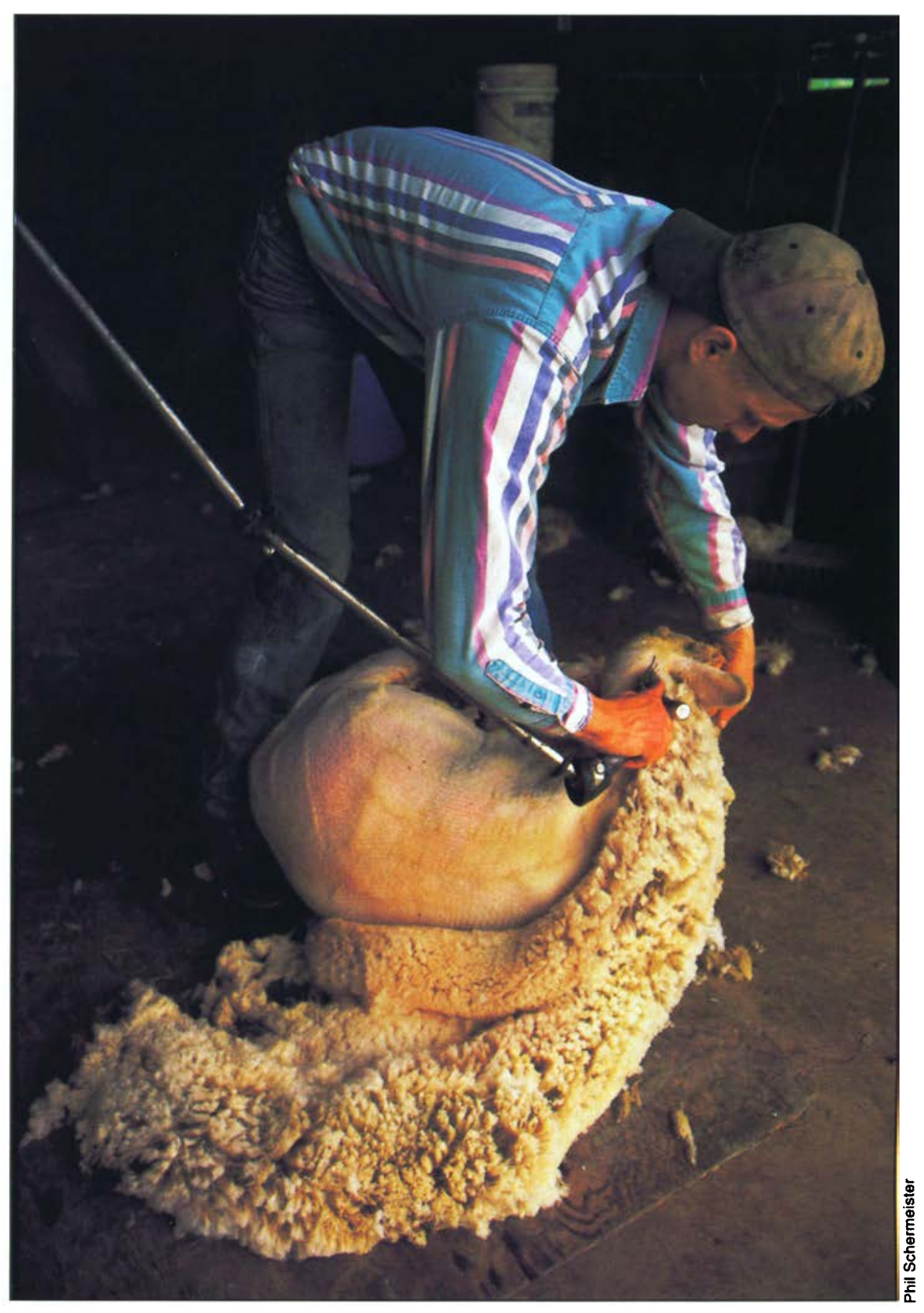

Animal-feed seeds engineered with higher levels of sulfur-containing amino acids could improve wool growth in sheep.

classes of phytochemicals that offer specific health benefits. Fruits and vegetables are a major source of beneficial phytochemicals (Dellapenna 1999).

Phytochemical families with clearly beneficial health properties include glucosinolates found in the brassica vegetables including broccoli; carotenoids, such as the tomato fruit pigment lycopene, found in many plant families; flavonoids, such as the isoflavones found in soybeans; and the anthocyanins and flavonols found in many fruits and vegetables.
Some foods containing consistently higher levels of these and other plant nutrients should be available through conventional breeding methods within 10 years. The natural variation that would provide the basis of health-enhanced varieties may be present already in breeding populations. Compared with traditional breeding strategies, the application of biotechnology to improve phytonutrient levels in whole foods is more difficult due to the complex array of potentially important chemicals and 

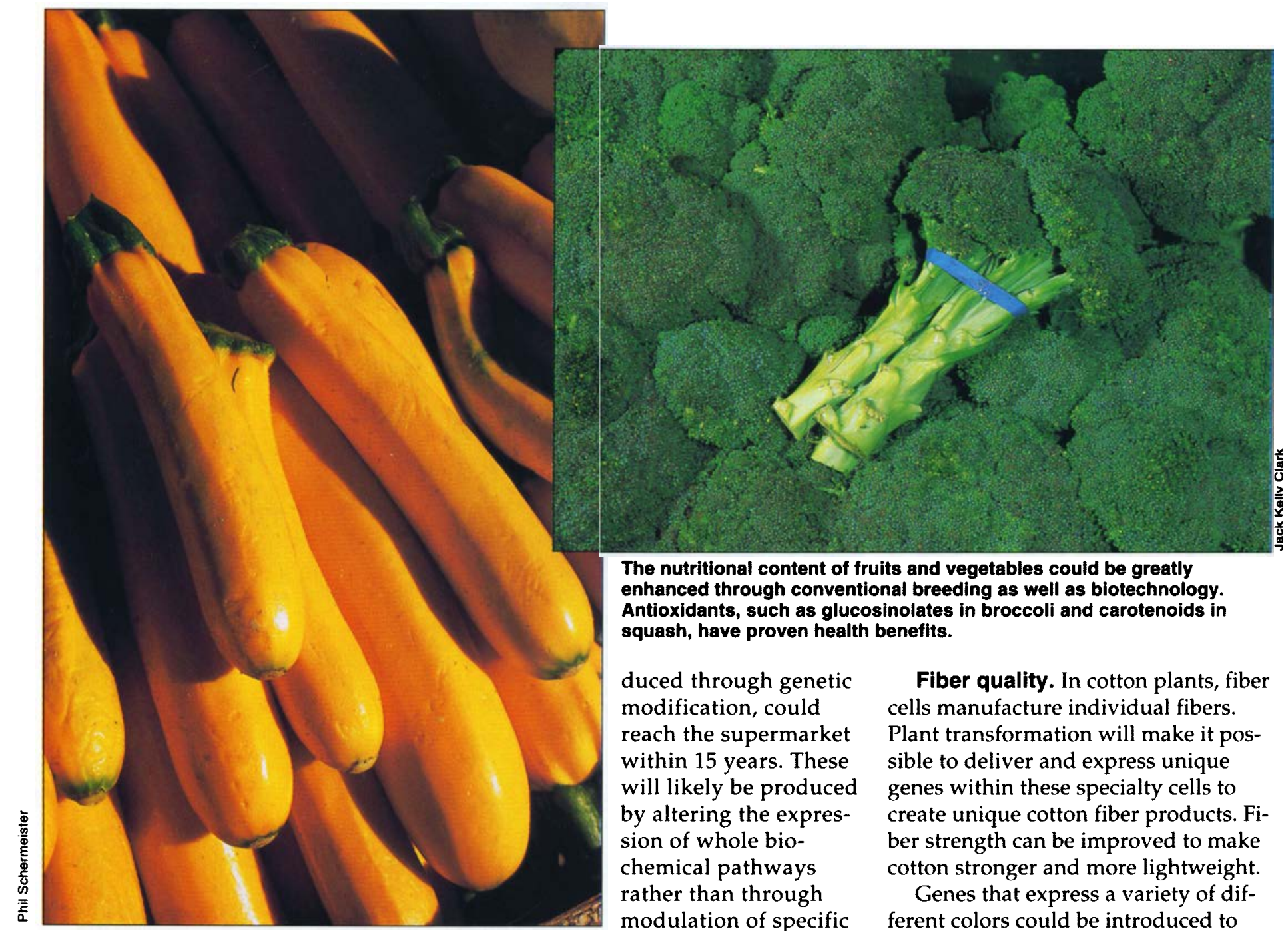

The nutritional content of fruits and vegetables could be greatly enhanced through conventional breeding as well as biotechnology. Antioxidants, such as glucosinolates in broccoli and carotenoids in squash, have proven health benefits.

duced through genetic modification, could reach the supermarket within 15 years. These will likely be produced by altering the expression of whole biochemical pathways rather than through modulation of specific enzymes.

Improved flavor is of major interest the complexity of the underlying biosynthetic pathways (Forkmann 1991).

Flavor and color. The ability to transgenically manipulate color intensity and hue was demonstrated more than 10 years ago (Meyer et al. 1987; Napoli et al. 1990). In flowers, the altered expression of the enzymes of flavonoid biosynthesis yielded novel floral pigmentation patterns. Such approaches have not been applied to fruits yet, but the potential exists.

Anthocyanins are the pigments responsible for color in many fruits, such as grapes and strawberries. Deeply colored fruits are generally more desirable to consumers. Further, anthocyanins and related flavonoids have antioxidant properties that reduce the risk of cardiovascular disease and cancer. Fruits with consistently higher levels of anthocyanins, pro- to consumers, but it does not receive significant attention from breeders, who work largely to improve production and durability during postharvest distribution. The complexity of flavor - which includes a balance between sweetness and acidity as well as the compounds that give products their characteristic taste - has discouraged the pursuit of biotechnological approaches to flavor improvement.

Biotechnological efforts to improve sweetness have met with little success so far. In some cases, an increase in sweetness leads to a decrease in size that is unacceptable in the marketplace. In addition, attempts to increase sweetness by expressing nonsugar, sweetness-enhancing proteins such as monellin have been frustrated because their compounds bind to cellular proteins and are subsequently not available to the sensory system.
Fiber quality. In cotton plants, fiber cells manufacture individual fibers. Plant transformation will make it possible to deliver and express unique genes within these specialty cells to create unique cotton fiber products. Fiber strength can be improved to make cotton stronger and more lightweight.

Genes that express a variety of different colors could be introduced to provide a greater range of naturally colored fibers. It should also be possible to enhance the quality of cotton fiber by synthesizing other polymeric materials to increase its strength, length and durability. The synthesis of unique polymeric materials in cotton could also enhance its thermal and elastic properties, creating a range of specialty fibers (Maliyakal and Greg 1996).

Seedlessness. Consumers clearly prefer seedless varieties of certain fruits; for example, seedless grapes and watermelons dominate the marketplace. Researchers are exploring novel strategies to convert seeded fruits to seedless versions more quickly and accurately than is possible with conventional breeding.

The application of molecular strategies for seedlessness could be of significant value to California grape and citrus growers. Grape breeders have been selecting for new seedless variet- 
ies for many years. Given the challenges inherent in breeding seedless grape varieties, it has been difficult to combine specific consumer and market preferences with seedlessness.

At least two different geneticengineering strategies are being attempted to convert seeded to seedless fruit. The first is to allow seed and fruit development to occur normally, but prevent formation of hard seed-coat layer (Koltunow et al. 1998). The second employs synthesis of the plant hormone auxin, which is triggered in ovules just before fertilization. The latter approach has been demonstrated effectively in eggplant (Rotino et al. 1997).

Ovule-specific auxin synthesis blocks fertilization and seed development and supports fruit development, which otherwise would require fertilization (Ficcadenti et al. 1999). The specific genes employed in eggplant may be applicable to related crops and the strategy could be adaptable to other fruits. These strategies for seedlessness will be applied to create new market niches and should be appearing in commercial produce within the next 10 years.

Shelf life and ripening. Two tomato products targeted at extending shelf life have met with limited success. One by Calgene reduces polygalacturonase activity to retard softening, while the other from DNA Plant Technology blocks ethylene synthesis to retard overall ripening. These products were not successful due to limitations in the quality of the base germplasm, the development of competitive nontransgenic products and the difficulty of obtaining premium prices when shelf life is not a primary consumer concern.

Nonetheless, other products with enhanced shelf life should reach the marketplace by the end of this decade. The increased popularity of ready-toeat and convenience foods will drive the need for products with improved shelf life. For example, sales of prepackaged lettuce have increased over the past 5 years. California agriculture is likely to take advantage of genetic modifications to reduce browning and oxidation and extend the shelf life of

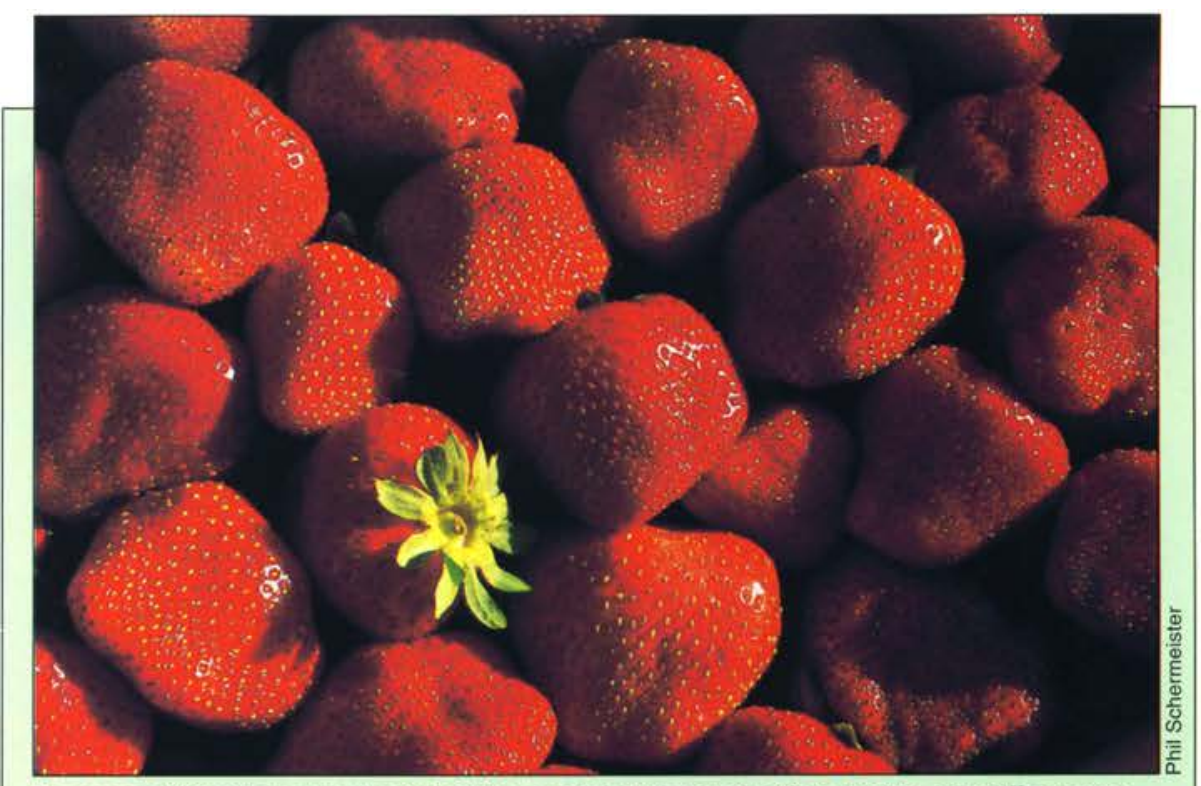

Because of their economic importance and high production costs, as well as ease of genetic manipulation, strawberries offer many opportunities for improvement. Research to control strawberry flowering and modify color, size and nutritional composition is under way.

\section{The future of strawberries}

Strawberry fields may be forever, but their look is likely to change dramatically over the next 25 years. From production systems to final product, the California strawberry industry will undergo a face-lift. Strawberries could incorporate many of the genetic modifications that we predict. Because of their economic importance and high production costs ( $\$ 35,000$ per acre), and ease in manipulating genetically, strawberries offer many opportunities for capturing value through modified varieties.

As regulatory mandates continue to reduce the use of chemicals with high environmental burdens, such as methyl bromide, strawberry varieties with improved resistance to a range of pathogens and pests could become the norm.

With flowering-control strategies resulting in earlier-fruiting varieties as well as varieties that produce more fruit over a shorter period, strawberries could be produced in more marginal areas (such as those with shorter growing seasons or poor soils) and with extended production periods. Given growers' need to reduce production costs - particularly for labor - to stay competitive in world markets, we can expect to see agricultural systems that give growers more precise control over the timing of fruit production.

Fruit quality will improve in a number of ways. Full development of strawberry fruit is linked to efficient fertilization and seed formation. Misshapen fruit can be produced by many varieties in conditions not conducive to fertilization. Strategies for seedlessness that eliminate the fertilization requirements could be applied to strawberries. Consumers may be able to purchase fruit of uniform size and shape. More uniformly colored varieties may also be available through modification of pathways that determine color formation and intensity, as will strawberries with enhanced nutritional composition.

In 2025, strawberry fruit will be a more reliable and better source of flavonoids and ascorbate than current varieties. Varieties with improved postharvest stability should also emerge by 2025 through control of fruit disease and novel ripening strategies, allowing new export opportunities for California strawberries. 

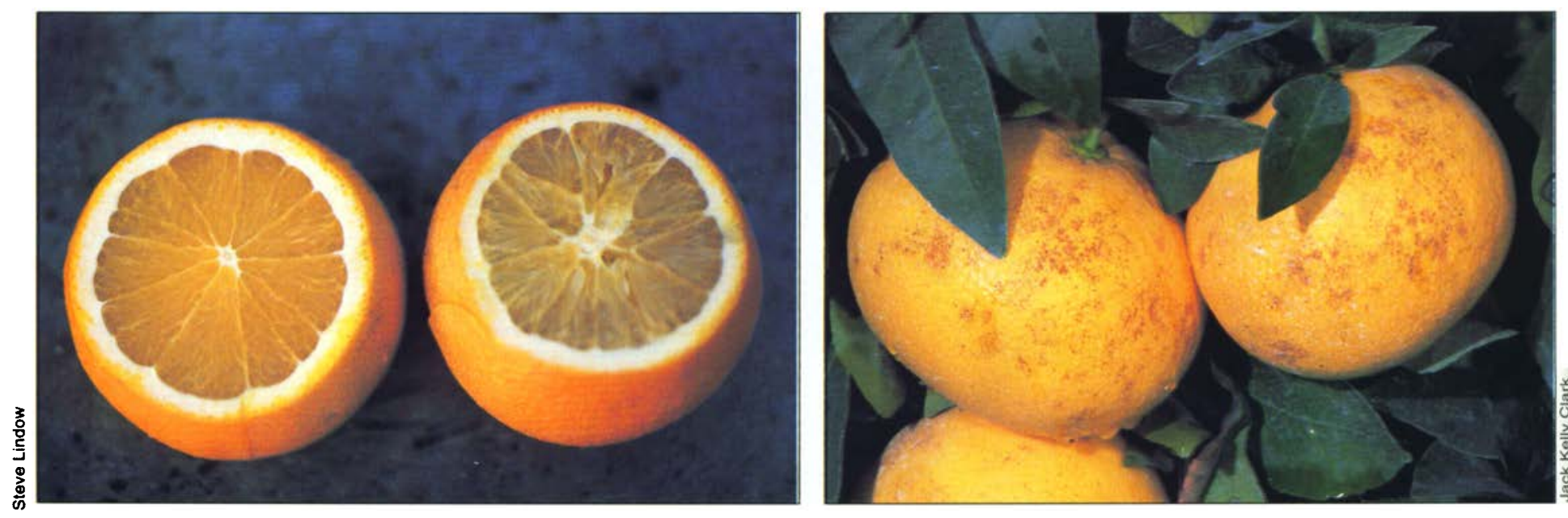

Some fruits and vegetables are highly susceptible to cold damage. The development of frost-resistant varieties could extend growing ranges and reduce production costs. Left, Severe frost damages orange pulp and lettuce leaves. Above, Grapefruit rinds stipple in prolonged cold, wet weather.

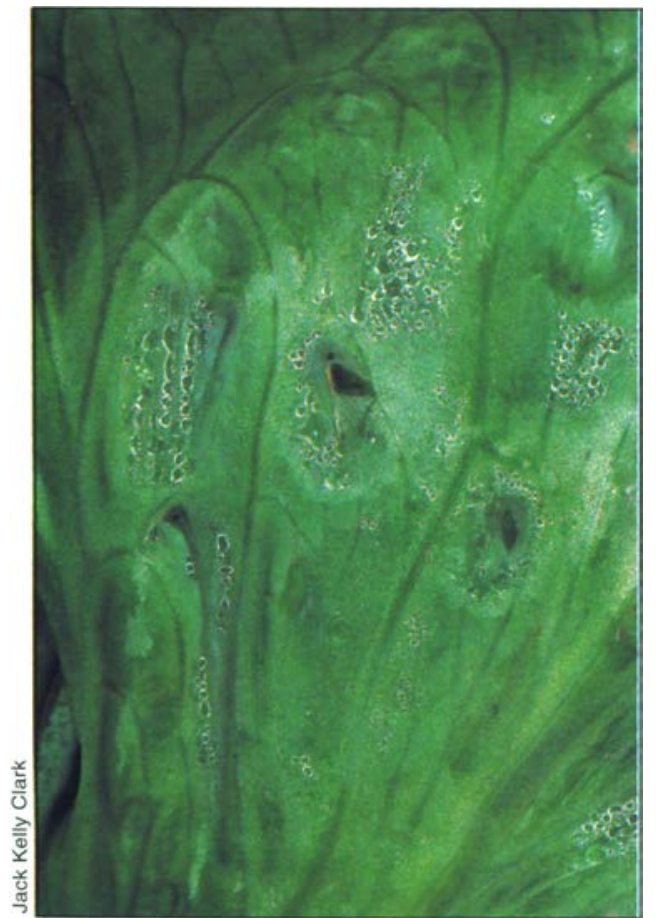

prepackaged lettuce. Researchers are also exploring approaches to retard wilting and yellowing in leafy green vegetables. The targeted expression of cytokinins to senescing leaves has shown promise as a route to extending shelf life of leafy vegetables (Ori et al. 1999).

In the case of fruits such as strawberries, extended shelf life could be obtained not only through modification of fruit texture and ripeness but also through reduced fruit disease susceptibility, all current targets of the agricultural biotechnology industry.
Botrytis gray mold is the principal postharvest disease limiting shelf life of strawberries produced in California. Researchers are evaluating a number of strategies to target this pathogen, including expression of proteins and secondary metabolites with antifungal activity, and activation of the plant's natural defenses. The pervasive gray mold pathogen will be a difficult challenge, but varieties with enhanced tolerance and longer shelf life should begin appearing in 10 years.

\section{Productivity and yields}

Early flowering. Thanks to the weed Arabidopsis thaliana, the favored experimental plant, much has been learned about the regulation of flower formation (Koornneef et al. 1998). Plants flower both in rhythm with their own internal development and in response to external signals such as temperature and day length.

For many herbaceous crop plants, altering the expression of genes identified principally through studies of Arabidopsis can produce a range of altered flowering patterns. These include neutral patterns (from previously day-length-dependent flowering time), flowering earlier or later in the growing season or changing the number of flowers per plant. Altered patterns can be obtained by altered ex- pression of genes such as constans and terminal flower, among others. Novel patterns can be exploited to secure better market opportunities or increase seasonal yields.

When foliage is the only desired product, the plant can be engineered not to flower at all in production fields, while producing seed efficiently in seed fields.

As the tools of genomics and genetic engineering are applied, we envision lettuce that does not flower in the fields, earlier-flowering strawberries and easier production of day-neutral strawberries. The power of this technology was demonstrated through chemical control of flowering in Arabidopsis (Simon et al. 1996).

Nature's own engineering has provided farmers with determinate-type tomatoes that differ from indeterminate types only through the mutation of a single gene, previously identified in Arabidopsis to control flower formation (Pnueli et al. 1998).

Controlling fruit formation in the many perennial crops grown in California will be challenging, as flowering is generally determined not by flower formation but by the release from dormancy. Scientists are studying the genetics and physiology of dormancy control. The application of genomic tools to dormancy in model 
crops should yield breakthroughs. Within 25 years, orchards may be planted with varieties that allow farmers to control the timing of fruit production.

Self-incompatibility. Selfincompatibility is a widespread mechanism in flowering plants that prevents self-fertilization, requiring the presence of pollinator plants to ensure cross-pollination for fruit production (Kao and McCubbin 1996). The predominant cause of self-incompatibility is the inability of the plant's pollen grains to successfully fertilize the ovary of the plant. This takes place either on the surface of the stigma or within the style; as a result, pollen grains either do not germinate or abort partially down the style. This latter type of incompatibility is a genetic trait encoded by a specific set of genes in the S-locus, referred to as S-alleles.

Unless the plant is cross-pollinated, seed and fruit production will be reduced. The S-alleles encode SRNase proteins are present in stylar tissue and are responsible for the inactivation of self-pollen tubes. Expression of antisense cDNA for these S-alleles in the style tissues can eliminate this problem allowing the plant to become self-fertile as long as there are no other anatomical problems with the flower that would interfere.

Self-pollination could greatly reduce the cost associated with ensuring adequate pollination of plants by bees or by hand. Self-pollination conserves land resources because pollinator plants do not need to be planted and there is more consistency in yield under variable environmental conditions. (Rain, for example, can interfere with bee pollination.) Self-pollination could have an impact on productivity. In the case of transgenic nut crops, it would allow the segregation of the transgenes, which would influence quality of the harvested nuts in a greater proportion due to genetic segregation.

Stress tolerance. The geographical distribution of growing regions is largely determined by climate, because drought and freezing tempera- tures stifle crop quality and productivity. Citrus crops are particularly susceptible to frost, so they are planted in the warmer microclimates of California and Florida. Traditional plantbreeding methods have had limited success in inducing drought and frost tolerance, even in plants. like wheat with active breeding programs. The freezing tolerance of the best wheat cultivars is essentially the same as those developed in the early part of the 20th century.

Development of frost- and drought-resistant cultivars

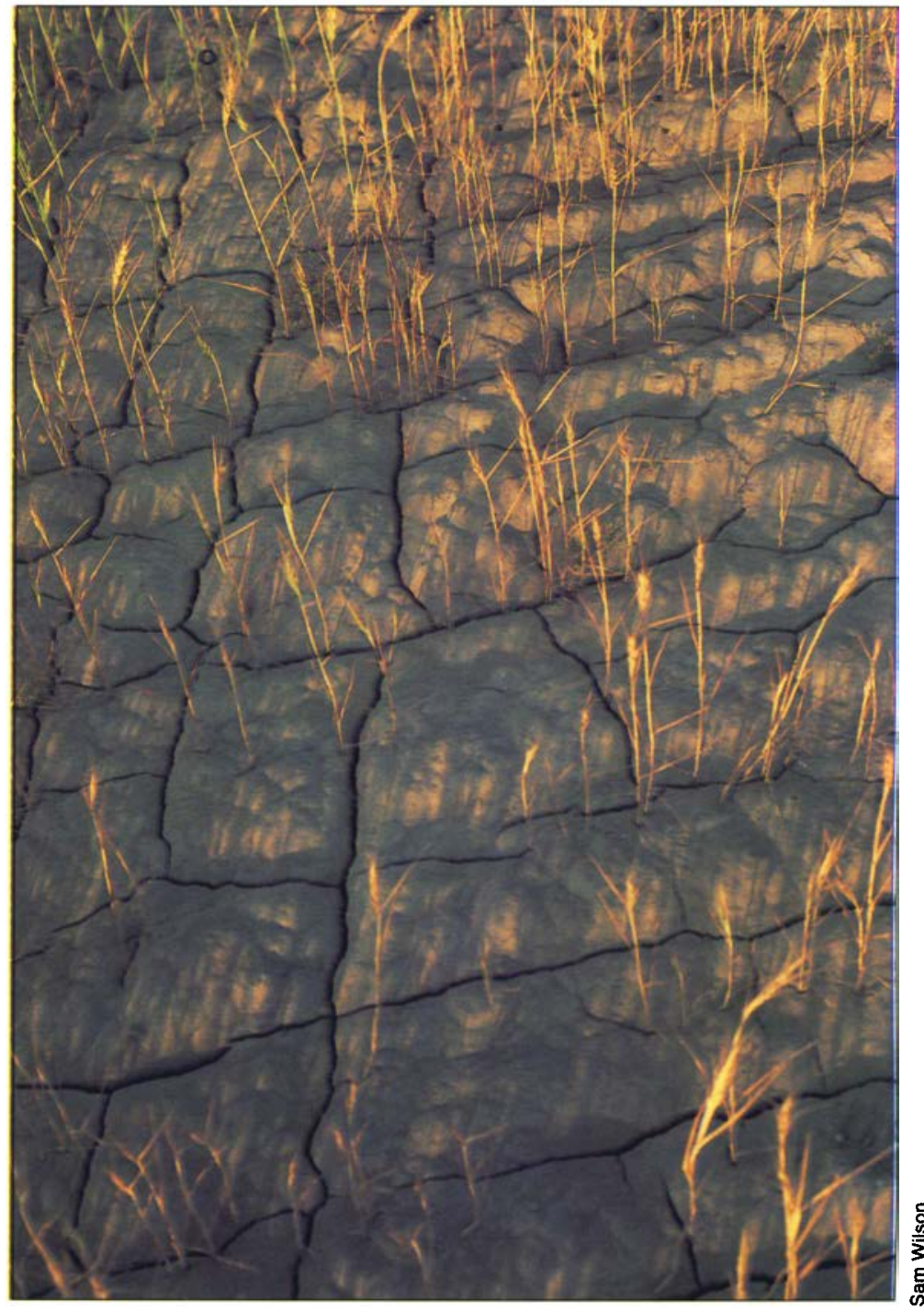

could allow greater flexibility in production areas, lower production costs and water conservation. Scientists working in this arena of plant technology strive to improve the ability of plants to withstand adverse environments. The major problem faced by plants growing in adverse environments is desiccation or drying at the cellular level, leading to the formation of free radicals that damage cellular organelles, disrupting cell function and ultimately killing the plant. This disruption of cellular activity can be prevented by engineering plants to synthesize specific compounds that stabilize cellular structures and contents as they desiccate, preventing free-radical oxidative damage (Smirnoff 1998). The development of plants with enhanced resistance to environmental stress could result in the
Drought-resistant crops could help to preserve water. Genetic-engineering research has focused on preventing desiccation at the cellular level.

cultivation of more tropical crops in temperate regions, such as growing mangoes in California.

\section{Medicinal applications}

By 2025 we could see plants used to synthesize a variety of medically active proteins, such as peptide hormones and therapeutic proteins like antibodies and bioactive peptides for the treatment of diseases (Rainer et al. 1999). These specialized crops would be planted in small acreages. The plant-based production of therapeutic proteins avoids many of the problems associated with animal-based production systems, such as high costs and contamination by viruses and hormones. 
For example, single-chain antibodies may be produced as diagnostic tools and for therapy. These could be generated efficiently in plants, and oral vaccines may also be synthesized in fruit or nut tissue.

As more is understood about oral vaccines, plants could be modified to prevent diseases such as hepatitis and influenza (Kapusta et al. 1999). Additionally, plants may be a good choice for an entire generation of drug development, such as the synthesis of patient-specific antibodies to treat non-Hodgkin's lymphoma

(McCormick et al. 1999).

Plants may also be used as factories for the synthesis of a variety of proteins for myriad uses, including industrial enzymes for detergents, baking, textile manufacturing and waste treatment.

\section{Future in focus: Working together}

The value and quality of California agricultural products could be significantly enhanced over the next 25 years through the application of genomics and genetic engineering tools. The snapshot of the strawberry crop (see sidebar, p. 53) is one of many from the future photo album of California agriculture. Appearance, taste and nutritional quality, along with enhanced production control, are among the plant traits that could be improved.

Many of California's most important crops, such as grapes, nuts and tree fruits have slower experimental and production establishment cycles than the large acreage, annual crops of the Midwest. As a result, novel technologies could reach California more slowly.

While genetic engineering has the power to bring new value to California agriculture, this technology is still in its infancy commercially. Calgene introduced the first transgenic plant product in 1994 (see p. 6), while the first major transgenic row crops were introduced in 1996. Farmer adoption of transgenic corn, soybean, cotton and canola varieties in the United States has occurred with almost unprecedented speed. Our projections assume continuing rapid adoption of transgenics, despite significant controversy surrounding their use in Europe and Japan, and to a lesser extent in the United States. It is possible that farmers or consumers will reject this technology broadly. More time may be necessary than we have anticipated for the science to deliver on its promise. Particularly for minor crops, which offer lower potential returns on investments, the technology may be slower to penetrate the marketplace.

We remain optimistic, however, that modern genetic technologies will be adopted, as the potential for both environmental and consumer benefits is large and regulatory agencies provide a level of oversight commensurate with the potential risks. Given the competitive pressures on California agriculture, government, university scientists and crop industries must work together to ensure the application of important new technologies to California crops.

A. Dandekar is Professor, Department of Pomology, UC Davis, and N. Gutterson is Director of Research, DNA Plant Technology, Oakland. DNAP conducts research and development toward improving strawberry and banana varieties, vegetable varieties (in collaboration with Seminis Vegetable Seeds), and technologies for disease and pest resistance. It also has a number of collaborative research relationships with $U C$ scientists.

\section{References}

Chrispeels MJ, Sadava DE. 1994. Human population growth: Lessons from demography. In: Plants, Genes, and Agriculture. Boston, MA: Jones and Bartlett. p 13-24.

Dellapenna D. 1999. Nutritional genomics: Manipulating plant micronutrients to improve human health. Science 285:375-9.

Ficcadenti N, Sesili S, Pandolfini T, et al. 1999. Genetic engineering of parthenocarpic fruit development in tomato. Molec Breeding 5:463-70.

Forkmann G. 1991. Flavonoids as flower pigments: The formation of the natural spectrum and its extension by genetic engineering. Plant Breeding 106:1-26.

Heyer AG, Lloyd JR, Kossmann J. 1999. Production of modified polymeric carbohydrates. Curr Opin Biotech 10:169-74.
Kao, T-H, McCubbin G. 1996. How flowering plants discriminate between self and nonself pollen to prevent inbreeding. Proceedings of the National Academy of Sciences USA 93:12059-65.

Kapusta J, Modelska A, Figlerowicz M, et al. 1999. A plant derived edible vaccine against hepatitis $B$ virus. FASEB $J$

13:1796-9.

Koltunow AM, Brennan P, Bond JE, Barker SJ. 1998. Evaluation of genes to reduce seed size in Arabidopsis and tobacco and their application to citrus. Molec Breeding 4:235-51.

Koornneef M, Alonso-Blanco C, Peeters AJM, Soppe W. 1998. Genetic control of flowering time in Arabidopsis. Annual Rev. Plant Physiol and Plant Molec Biol 49:345-70.

Maliyakal JE, Greg K. 1996. Metabolic pathway engineering in cotton: Biosynthesis of polyhydroxybutyrate in fiber cells. Proceedings of the National Academy of Sciences USA 93:12768-73.

Martin GB. 1998. Gene discovery for crop improvement. Curr Opin Biotech 9:220-6.

McCormick AA, Kumagai MH, Hanley K, et al. 1999. Rapid production of specific vaccines for lymphoma by expression of the tumor-derived single chain Fv epitopes in tobacco plants. Proceedings of the National Academy of Sciences USA 96:703-8.

Meyer P, Heidmann I, Forkmann G, Saedler H. 1987. A new petunia flower colour generated by transformation of a mutant with a maize gene. Nature 330:677-8.

Napoli C, Lemieux C, Jorgensen R. 1990. Introduction of a chimeric chalcone synthase gene into petunia results in reversible co-suppression of homologous genes in trans. The Plant Cell 2:279-89.

Ori N, Juarez MT, Jackson D, et al. 1999. Leat senescence is delayed in tobacco plants expressing the maize homeobox gene knotted1 under the control of a senescenceactivated promoter. The Plant Cell 11:107380.

Pnueli L, Carmel-Goren L, Hareven D, et al. 1998. The self-pruning gene of tomato regulates vegetative to reproductive switching of sympodial meristems and is the orthology of CEN and TFL1. Development 125:197989.

Rainer F, Juergen D, Ulrich C, et al. 1999. Towards molecular farming in the future: Moving from diagnostic protein and antibody production in microbes to plants. Biotech Appl Biochem 30:101-8.

Rotino GL, Perri E, Zottini M, et al. 1997. Genetic engineering of parthenocarpic plants. Nat Biotech 151:398-401.

Simon R, Igeno MI, Coupland G. 1996. Activation of floral meristem identity genes in Arabidopsis. Nature 384:59-62.

Smirnoff N. 1998. Plant resistance to environmental stress. Curr Opin Biotechnol 9:214-9.

Topfer R, Martini N, Schell J.,1995. Modification of plant lipid synthesis. Science 268:681-6. 